\title{
Brane cosmological evolution with a general bulk matter configuration
}

\author{
Pantelis S. Apostolopoulos* and Nikolaos Tetradis ${ }^{\dagger}$ \\ University of Athens, Department of Physics, Nuclear and Particle Physics Section, \\ Panepistimiopolis, Zografos 15771, Athens, Greece.
}

(Dated: June 4, 2018)

\begin{abstract}
Using a fully covariant treatment for the description of the bulk geometry, we study the brane cosmological evolution in the presence of a smooth bulk matter distribution. We focus on the case of a Friedmann-Robertson-Walker (FRW) brane, invariantly characterized by the existence of a six-dimensional group of isometries acting on 3D spacelike orbits. With a FRW brane, the bulk geometry can be regarded as the 5D generalization of the inhomogeneous orthogonal family of Locally Rotationally Symmetric (LRS) spacetimes. We show that, for any bulk matter configuration, the expansion rate on the brane depends only on the covariantly defined comoving mass $\mathcal{M}$ of the bulk fluid within a radius equal to the average length scale of the $3 \mathrm{D}$ spacelike hypersurfaces of constant curvature. This unique contribution incorporates the effects of the 5D Weyl tensor and the projected tensor related to the bulk matter, and gives a transparent physical picture that includes an effective conservation equation between the brane and the bulk matter.
\end{abstract}

\section{INTRODUCTION}

The possibility that our Universe is identified with a 4D hypersurface (3-brane) embedded in a higherdimensional bulk space allows for interesting novel features in the cosmological evolution as perceived by a brane observer. Consistency with the standard cosmological expansion can be achieved if the extra dimensions are "warped" in a way that low-energy gravitons are localized on the brane 1]. This requires a negative cosmological constant in the bulk. The presence of matter localized on the brane induces a cosmological expansion. The 3-brane must also have a tension that balances the effect of the negative cosmological constant on the expansion. For low energy densities, the effective Friedmann equation on the brane has the standard form 2, 3, 4, 5]. Several features and variations of this scenario have been consider. (For recent reviews see 6, 7].) We are interested in the effects arising from the presence of additional matter in the bulk, such as gravitational radiation emitted or absorbed by the brane [8, 9], or bulk fields [10]. The possible energy exchange between the brane and the bulk can induce important modifications of the cosmological evolution 11, 12, 13].

In this work we would like to address the problem of the effect of the bulk matter on the brane cosmological evolution in a general way. For this reason we employ the covariant formalism [5, 7] that permits us to describe the evolution without reference to a particular local system of coordinates. We assume that the spatial part of the brane metric is maximally symmetric, in agreement with the homogeneity and isotropy of the observed Universe at large scales. We refer to it as a FriedmannRobertson-Walker (FRW) brane. As a consequence, the bulk geometry can be seen as the $5 \mathrm{D}$ generalization of the

\footnotetext{
*Email address: papost@phys.uoa.gr

${ }^{\dagger}$ Email address: ntetrad@cc.uoa.gr
}

inhomogeneous orthogonal Locally Rotationally Symmetric $(L R S)$ spacetimes [14]. Our main result is that the symmetry of the FRW brane constraints the form of the bulk contribution to the brane evolution. The effective Friedmann equation contains only one term originating in the bulk matter. The physical quantity affecting the brane evolution is the covariantly defined comoving mass of the bulk fluid within a radius equal to the average length scale of the 3D spacelike hypersurface (essentially the value of the scale factor).

In the following section we summarize the known results for the bulk-brane dynamics within the covariant formulation. In section III we concentrate on the case of a FRW brane. We invariantly characterize the bulk geometry in terms of the unique preferred spacelike direction that represents the local (axis) of symmetry. This naturally emerges from the assumption of the rotational symmetry of the $5 \mathrm{D}$ bulk. In section IV we express covariantly the electric part of the Weyl tensor in terms of the irreducible parts of a general bulk energy-momentum tensor with respect to a generic bulk observer $u^{A}$. The comoving mass appears as a first integral of the constraint equations satisfied by the shear tensor and the electric part of the 5D Weyl tensor. As two concrete and illustrative examples, we apply the above considerations to the Gauss-normal coordinate system in which the brane is located at a fixed value of the fourth spatial coordinate (brane comoving system), and to the coordinate system adapted to the bulk observers $u^{A}$ (bulk comoving system) in which the brane is moving. This approach shows the equivalence of these points of view for any bulk matter configuration. In section $\mathrm{V}$ we use these results in order to derive the effective Friedmann and Raychaudhuri equations governing the cosmological evolution on the FRW brane. A combination of these equations has the form of a conservation equation involving the brane energy density and an effective density originating in the bulk matter. Our conclusions are given in section VI.

Throughout this work we use the following index con- 
ventions: bulk $5 \mathrm{D}$ indices are denoted by capital latin letters $A, B, \ldots=0,1,2,3,4$ and greek letters denote spacetime indices $\alpha, \beta, \ldots=0,1,2,3$.

\section{BRANE DYNAMICS WITH MATTER IN THE BULK}

We are interested in cases with a matter component in the bulk in addition to the negative cosmological constant. We consider an action of the form

$$
\begin{aligned}
S= & \int d^{5} x \sqrt{-g}\left(\Lambda+M^{3} R+\mathcal{L}_{\mathrm{BULK}}^{\text {mat }}\right)+ \\
& +\int d^{4} x \sqrt{-g}\left(-V+\mathcal{L}_{\mathrm{BRANE}}^{\text {mat }}\right),
\end{aligned}
$$

where $R$ is the curvature scalar of the five-dimensional bulk metric $g_{A B},-\Lambda$ the bulk cosmological constant $(\Lambda>0), V$ the brane tension, and $g_{\alpha \beta}$ the induced metric on the brane. The brane is assumed to be normal to the unit spacelike vector field $n^{A}$ that is tangential to the extra spatial dimension.

The Einstein Equations (EE) in the bulk take the usual form

$$
G_{B}^{A}=\frac{1}{2 M^{3}}\left(T_{B}^{A}+\Lambda \delta_{B}^{A}\right),
$$

where $T_{B}^{A}$ denotes the total energy-momentum tensor, i.e.

$$
T_{A B}=T_{A B}^{\mathrm{BULK}}+\delta(n)\left(T_{A B}^{\mathrm{BRANE}}+V g_{A B}\right) .
$$

The modified $4 \mathrm{D}$ EE are derived by assuming a $Z_{2}$ symmetry of the bulk around the brane and employing Israel's junction conditions [7, 16]

$$
G_{\alpha \beta}=-3 \lambda g_{\alpha \beta}+\frac{V}{24 M^{6}} T_{\alpha \beta}+\frac{1}{4 M^{6}} \mathcal{S}_{\alpha \beta}-\mathcal{E}_{\alpha \beta}+\frac{1}{3 M^{3}} \mathcal{F}_{\alpha \beta},
$$

where

$$
\begin{gathered}
\mathcal{S}_{\alpha \beta}=\frac{1}{2} T T_{\alpha \beta}-\frac{1}{4} T_{\alpha \gamma} T_{\beta}^{\gamma}+\frac{3 T_{\gamma \delta} T^{\gamma \delta}-T^{2}}{24} g_{\alpha \beta} \\
\mathcal{F}_{\alpha \beta}=T_{A B}^{\mathrm{BULK}} g^{A}{ }_{\alpha} g^{B}+\left(T_{A B}^{\mathrm{BULK}} n^{A} n^{B}-\frac{T^{\mathrm{BULK}}}{4}\right) g_{\alpha \beta} \\
\mathcal{E}_{\alpha \beta}=\mathcal{E}_{A B} g_{\alpha}^{A} g^{B}{ }_{\beta}=C_{A C B D} n^{C} n^{D} g^{A}{ }_{\alpha} g^{B}
\end{gathered}
$$

and $\lambda=\left(V^{2} / 12 M^{3}-\Lambda\right) / 12 M^{3}$ is the effective cosmological constant.

From the EE (4) we observe that, apart from the terms quadradic to the brane energy-momentum tensor, there exist two additional terms corresponding to: a) the projection of the $5 \mathrm{D}$ Weyl tensor $\mathcal{E}_{\alpha \beta}$ and b) the projected (normal to $n^{A}$ ) tensor $\mathcal{F}_{\alpha \beta}$ that contains the bulk matter contribution. Since both tensors are 5D objects we conclude that both induce bulk effects on the brane. In the case of an empty bulk, the 5D contributions on the brane are coming from the non-local effects of the free gravitational field incorporated in $\mathcal{E}_{\alpha \beta}$ (5D bulk gravitons). As we shall see in the subsequent sections, when the bulk is non-empty, the tensor $\mathcal{E}_{\alpha \beta}$ is expressed in terms of the bulk matter configuration. Therefore it is clear that, in order to analyze the cosmological evolution on the brane, we must take into account the contribution of the bulk energy-momentum tensor.

Formally any type of bulk matter can be covariantly described by an energy-momentum tensor decomposed with respect to the cosmological (brane) observers $\tilde{u}_{\alpha} \equiv$ $g_{\alpha}^{A} \tilde{u}_{A}$ as

$$
T_{A B}^{\mathrm{BULK}}=\bar{\rho} \tilde{u}_{A} \tilde{u}_{B}+\bar{p} \tilde{h}_{A B}+2 \bar{q}_{(A} \tilde{u}_{B)}+\bar{\pi}_{A B} .
$$

The energy density $\bar{\rho}$, isotropic pressure $\bar{p}$, energy flux vector $\bar{q}_{A}$ and anisotropic pressure tensor $\bar{\pi}_{A B}$, measured by the brane observers, are covariantly defined as

$$
\begin{aligned}
\bar{\rho} & =T_{A B} \tilde{u}^{A} \tilde{u}^{B}, \bar{p}=\frac{1}{4} T_{A B} \tilde{h}^{A B}, \bar{q}_{A}=-\tilde{h}_{A}^{C} T_{C D} \tilde{u}^{D}, \\
\bar{\pi}_{A B} & =\tilde{h}_{A}^{C} \tilde{h}_{B}^{D} T_{C D}-\frac{1}{4}\left(\tilde{h}^{C D} T_{C D}\right) \tilde{h}_{A B},
\end{aligned}
$$

where $\tilde{h}_{A B}=g_{A B}+\tilde{u}_{A} \tilde{u}_{B}$ is the projection tensor perpendicularly to $\tilde{u}^{A}$.

With these identifications the conservation equation on the brane, coming from the twice contracted Bianchi identities, implies []

$$
T_{\alpha \beta}{ }^{; \beta}=-2\left[\left(\bar{q}_{C} n^{C}\right) \tilde{u}_{\alpha}+\bar{\pi}_{A B} n^{B} g_{\alpha}^{A}\right] .
$$

This shows that in general the brane matter is not conserved, but there is energy exchange (outflow or inflow) between the brane and bulk, that depends on the character of the vector field $\left(\bar{q}_{C} n^{C}\right) \tilde{u}_{\alpha}+\bar{\pi}_{A B} n^{B} g_{\alpha}^{A}$ involving the energy flux vector $\bar{q}_{A}$ and the bulk anisotropic stress vector $\bar{\pi}_{A B} n^{B}$.

\section{AN INVARIANT DESCRIPTION OF THE BULK GEOMETRY WITH A FRW BRANE}

For a FRW brane the 3D hypersurfaces $\mathcal{D}$ normal to the prolongated cosmological observers $\tilde{u}_{A}$ are maximally symmetric. In geometrical terms this means that the full 5D space admits a six-dimensional multiply transitive group of isometries acting on 3D spacelike orbits. This in turn implies the existence of a one-parameter continuous isotropy subgroup. Consequently, the bulk geometry with a FRW brane can be seen as a 5D generalization of the inhomogeneous orthogonal family of Locally Rotationally Symmetric (LRS class II) spacetimes 14].

Let us examine the kinematics and dynamics of the bulk from the point of view of a bulk observer $u^{A}$ $\left(u^{A} u_{A}=-1\right.$ and $\left.u_{A} \neq \tilde{u}_{A}\right)$. The deformation of the 
timelike congruence $u^{A}$ is covariantly described by the irreducible $1+4$ threading of its first derivatives in the standard way 15

$$
u_{A ; B}=\Sigma_{A B}+\frac{\Theta}{4} h_{A B}+\Omega_{B A}-\dot{u}_{A} u_{B}
$$

where $\Sigma_{A B}=\left(h_{A}^{K} h_{B}^{L}-\frac{1}{4} h^{K L} h_{A B}\right) u_{(K ; L)}, \Theta=u_{; A}^{A}$, $\Omega_{B A}=u_{[A ; B]}+\dot{u}_{[A} u_{B]}$ and $\dot{u}^{A}=u_{; B}^{A} u^{B}$ are the rate of shear tensor, the rate of expansion scalar, the vorticity tensor and the acceleration of the observers $u^{A}$, respectively, and $h_{A B}=g_{A B}+u_{A} u_{B}$ is the projection operator perpendicularly to $u^{A}$. We assume that the timelike congruence $u^{A}$ is irrotational, i.e. $\Omega_{A B}=0$. This is always possible within the geometry we are considering.

Regarding the dynamics, the matter content of the bulk is described by the energy-momentum $T_{A B}^{\mathrm{BULK}}$, which with respect to the observers $u^{A}$ can be written as

$$
T_{A B}^{\mathrm{BULK}}=\rho u_{A} u_{B}+p h_{A B}+2 q_{(A} u_{B)}+\pi_{A B} .
$$

The dynamical quantities measured by the bulk observers are defined in a similar manner as in (9).

Because of the LRS bulk geometry, there exists a preferred spacelike direction $e^{A}$ representing the (local) axis of symmetry with respect to which all the geometrical, kinematical and dynamical quantities are invariant. It is natural to select $e^{A}$ to be the normal to the timelike congruence of the bulk observer $u^{A}$, i.e. $u^{A} e_{A}=0$. As a result all the spacelike vector or tensor fields perpendicular to $u^{A}$ can be written in terms of $e^{A}$.

In complete analogy with [14] we introduce the unique spacelike and trace-free tensor

$$
e_{A B}=e_{(A B)}=\frac{1}{3}\left(4 e_{A} e_{B}-h_{A B}\right)=h_{A B}-\frac{4}{3} \Pi_{A B}
$$

where the projection operator $\Pi_{A B}$ is defined as

$$
\Pi_{A B} \equiv g_{A B}+u_{A} u_{B}-e_{A} e_{B}=h_{A B}-e_{A} e_{B}
$$

and has the usual projection properties

$$
\Pi_{A}^{A}=3, \quad \Pi_{C}^{A} \Pi_{B}^{C}=\Pi_{B}^{A}, \quad \Pi_{B}^{A} e^{B}=\Pi_{B}^{A} u^{B}=0
$$

i.e. it projects normally both to $u^{A}$ and $e^{A}$. Essentially $\Pi_{A B}$ is used for the $1+1+3$ decomposition of the tangent space. In the particular case of the FRW brane it corresponds to the induced metric of the 3D hypersurfaces of constant curvature.

Using the above definitions it will be convenient to express every covariantly defined spacelike and traceless tensor $\Lambda_{A B}$ as

$$
\Lambda_{A B}=\sqrt{\frac{3}{2}} \Lambda e_{A B}
$$

where $\Lambda^{2}=\Lambda_{A B} \Lambda^{A B} / 2$ is the norm of the trace-free tensor $\Lambda_{A B}$.

\section{THE WEYL RADIATIVE ENERGY}

Our aim in this section is to express the scalar

$$
\mathcal{E}=C_{A C B D} \tilde{u}^{A} n^{C} \tilde{u}^{B} n^{D}
$$

in terms of the bulk matter. It will prove easier to compute the scalar $E=C_{A C B D} u^{A} e^{C} u^{B} e^{D}$ with respect to the bulk observers $u^{A}$ and the preferred spatial direction $e^{A}\left(e^{A} u_{A}=0\right)$. It can be shown easily, by performing a $1+4$ decomposition of $\tilde{u}^{A}$ and $n^{A}$ w.r.t. $u^{A}$ and $e^{A}$, that $\mathcal{E}=E$.

Using the Ricci identities for $u^{A}$, the normal projection of its trace and the energy-momentum decomposition (12) gives the generalized constraint equation for the shear tensor of the (irrotational) bulk observers

$$
D_{K} \Sigma_{A}^{K}=\frac{3}{4} D_{A} \Theta-\frac{1}{2 M^{3}} q_{A} .
$$

We have employed here the fully projected (perpendicular to $u^{A}$ ) covariant derivative $D_{A}$ which, for any tensor $P^{A B \ldots}{ }_{I J \ldots}$, is defined as

$$
D_{L} P^{A B \ldots}{ }_{I J \ldots} \equiv h_{R}^{A} h_{S}^{B} \ldots h_{I}^{T} h_{J}^{X} \ldots h_{L}^{K}\left(P^{R S \ldots}{ }_{T X \ldots}\right)_{; K}
$$

where a semicolon ";" denotes the usual covariant derivative associated with the bulk metric $g_{A B}$.

The trace of Bianchi identities $R_{A B[C D ; E]}=0$ in a $5 \mathrm{D}$ space gives

$$
C_{A B C ; K}^{K}=\frac{4}{3}\left(R_{A[C ; B]}-\frac{1}{8} g_{A[C} R_{; B]}\right)
$$

We note that, in contrast to the $4 \mathrm{D}$ geometries, equation (20) is not equivalent to the full set of Bianchi identities. However, for the purposes of the present work it will be sufficient. In fact, the $1+4$ decomposition of (20) gives propagation (parallel to $u^{A}$ ) and constraint (normal to $u^{A}$ ) equations for the electric part $E_{A B}=C_{A C B D} u^{C} u^{D}$ of the Weyl tensor. From these we shall need only the latter. After a straightforward calculation we obtain the generalized constraint equation for the electric part of the 5D Weyl tensor 


$$
D_{K}\left(E^{K}{ }_{A}+\frac{1}{2 M^{3}} \frac{2}{3} \pi^{K}{ }_{A}\right)=\frac{1}{2 M^{3}} \frac{1}{2} D_{A} \rho+\frac{1}{2 M^{3}} \frac{2}{3} \Sigma^{K}{ }_{A} q_{K}-\frac{1}{2 M^{3}} \frac{1}{2} q_{A} \Theta-h_{A}^{B} C^{K}{ }_{L C B} \Sigma^{C}{ }_{K} u^{L}
$$

where we have incorporated the EE (2) away from the brane.

Denoting with a prime the spatial derivative with respect to the preferred direction $e^{A}$, and using the fact that $\Sigma_{B}^{A}$ and $\pi_{B}^{A}$ are spacelike traceless tensors, equations (16), (18) and (21) give

$$
\frac{1}{\ell^{4}}\left\{\left[E+\frac{1}{2 M^{3}} \frac{2}{3}\left(\pi_{A B} e^{A} e^{B}\right)\right] \ell^{4}\right\}^{\prime}=\frac{1}{2 M^{3}} \frac{1}{2} \rho^{\prime} .
$$

We have introduced the average length scale function $\ell$ of the spacelike congruence $e^{A}$ defined as $D_{A} e^{A} \equiv 3 \ell^{\prime} / \ell$. Essentially the quantity $D_{A} e^{A}$ corresponds to the overall expansion of the spacelike congruence as measured in the rest space of the observers $u^{A}$. Therefore, $\ell$ represents the radius of the $3 D$ spatial slices $\mathcal{D}$.

Equation (14) implies

$\pi_{A B} e^{A} e^{B}=\left(T_{A B}^{\mathrm{BULK}}-p h_{A B}\right) e^{A} e^{B}=T^{\mathrm{BULK}}+\rho-3 p_{\perp}-p$.

The pressure $3 p_{\perp} \equiv \Pi^{A B} T_{A B}^{\mathrm{BULK}}$ is perpendicular to both $u^{A}, e^{A}$ and corresponds to the isotropic pressure measured by the bulk observers.

Integrating (22) and using equation (23) we finally get

$$
\mathcal{E}=E=-\frac{1}{2 M^{3}}\left[\frac{1}{2}\left(T^{\mathrm{BULK}}-4 p_{\perp}\right)+\frac{\mathcal{M}}{\pi^{2} \ell^{4}}\right]
$$

where

$$
\mathcal{M}=\int_{0}^{\ell} 2 \pi^{2} \rho \ell^{3} d \ell+\mathcal{M}_{0}
$$

can be interpreted as the generalized comoving mass of the bulk fluid within radius $\ell$.

It would be useful to give some familiar examples by choosing specific (local) coordinate systems, in order to express equation (24) in analytic form. In particular, it is possible to discuss the brane evolution either in the Gauss-normal coordinate system in which the brane is located at a fixed value of the fourth spatial coordinate (brane comoving system), or in the coordinate system adapted to the bulk observers $u^{A}$ (bulk comoving system) in which the brane is moving [2, 3, 4].

In the latter case local coordinates can be found such that $u^{A}=n^{-1}(t, r) \delta^{A}{ }_{t}$ and the bulk metric is written in Schwarzschild coordinates as

$$
d s^{2}=-n^{2}(t, r) d t^{2}+b^{2}(t, r) d r^{2}+r^{2} d \Omega_{k}^{2}
$$

where $d \Omega_{k}^{2}$ is the metric of the $3 \mathrm{D}$ hypersurfaces $\mathcal{D}$ of constant curvature that is parametrized by $k=-1,0,1$.
Obviously the preferred spatial axis of symmetry is $\sim \partial_{r}$, and therefore $\ell \equiv r$. This implies that the integrated matter distribution between $r=0$ and some arbitrary radius $r$ is $\mathcal{M}=\int_{0}^{r} 2 \pi^{2} \rho r^{3} d r+\mathcal{M}_{0}$. The integration constant $\mathcal{M}_{0}$ is the contribution from the mass of a black hole located at $r=0$.

On the other hand, using Gauss-normal coordinates for the bulk with the brane located at a fixed value (e.g. $\eta=0$ ) of the fourth spatial coordinate, the metric is

$$
\begin{aligned}
d s^{2}= & \gamma_{\alpha \beta} d x^{\alpha} d x^{\beta}+d \eta^{2}= \\
& -\tilde{m}^{2}(\tilde{\tau}, \eta) d \tilde{\tau}^{2}+R^{2}(\tilde{\tau}, \eta) d \Omega_{k}^{2}+d \eta^{2} .
\end{aligned}
$$

In this case $\ell=R(\tilde{\tau}, \eta)$ represents the average length scale for distances between any pair of brane observers. It follows that, at the location of the brane $\eta=0$, the quantity $\ell$ corresponds to the scale factor of the FRW brane. The generalized comoving mass is $\mathcal{M}=$ $\int_{0}^{R} 2 \pi^{2} \rho R^{3} d R+\mathcal{M}_{0}=\int_{0}^{R} 2 \pi^{2} T_{A B}^{\mathrm{BULK}} u^{A} u^{B} R^{3} d R+\mathcal{M}_{0}$.

We conclude this section by pointing out that the equivalence between the two points of view has been shown for an empty bulk $\left(T_{A B}^{\mathrm{BULK}}=0\right)$. In this case the $5 \mathrm{D}$ metric is static and reduces to the well-known 5D AdSSchwarzschild with $\mathcal{M}=\mathcal{M}_{0}$ representing the black hole mass [4, 17, 18]. The present approach shows that this equivalence holds in general and irrespectively of the specific assumptions for the bulk matter distribution.

\section{THE INDUCED MODIFICATIONS OF THE BRANE EVOLUTION}

The invariant characterization of the FRW brane is the vanishing of the vorticity, shear and acceleration of the timelike congruence $\tilde{u}_{\alpha}$. Assuming a perfect fluid matter configuration on the brane, the energy-momentum tensor is written in terms of the energy density $\tilde{\rho}$ and the isotropic pressure $\tilde{p}$ as

$$
T_{\alpha \beta}=\tilde{\rho} \tilde{u}_{\alpha} \tilde{u}_{\beta}+\tilde{p} \tilde{h}_{\alpha \beta} .
$$

The conservation equation (10) can be split along and normally to $\tilde{u}^{\alpha}$. In this way we obtain the propagation equation for the energy density $\tilde{\rho}$

$$
\dot{\tilde{\rho}}+3 H(\tilde{\rho}+\tilde{p})=2 \bar{q}_{C} n^{C} .
$$

This equation shows that the rate of energy transfer between the bulk and the brane is controlled by the energy flux vector field $\bar{q}_{A}$ that appears in the decomposition of the bulk energy-momentum tensor according to (12). 
It should be noted that the normal part of (10) gives the spatial gradient of $\tilde{p}$

$$
h_{\alpha}^{\beta} \tilde{p}_{; \beta}=-2 \bar{\pi}_{A B} n^{B} g_{\alpha}^{A} .
$$

However, taking into account the relation (16), equation (30) implies the standard spatial homogeneity $h_{\alpha}^{\beta} \tilde{p}_{; \beta}=0$ of the isotropic pressure $\tilde{p}$.

The brane evolution can be studied by determining the generalized Friedmann and Raychaudhuri equations on the brane in the presence of bulk matter. These follow from the Gauss-Codazzi equations and the timelike part of the trace of the Ricci identities applied to the (irrotational, geodesic and shear-free) timelike congruence $\tilde{u}_{\alpha}$. They have the form

$$
\begin{gathered}
H^{2}=\frac{1}{3} R_{\alpha \beta} \tilde{u}^{\alpha} \tilde{u}^{\beta}-\frac{{ }^{3} R}{6}+\frac{R}{6} \\
\dot{H}=-H^{2}-\frac{1}{3} R_{\alpha \beta} \tilde{u}^{\alpha} \tilde{u}^{\beta},
\end{gathered}
$$

where $R_{\alpha \beta}=G_{\alpha \beta}-\frac{G}{2} g_{\alpha \beta}$ is the modified Ricci tensor of the brane, $3 H=\tilde{u}_{; \alpha}^{\alpha}$ the Hubble parameter, ${ }^{3} R$ the scalar curvature of the $3 \mathrm{D}$ hypersurfaces $\mathcal{D}$, and a dot denotes differentiation with respect to $\tilde{u}^{\alpha}$, i.e. $\dot{H}=H_{; \alpha} \tilde{u}^{\alpha}$.

Using equation (4) and the decomposition (8), equations (31) and (32) become:

$$
\begin{aligned}
H^{2} & =\lambda-\frac{{ }^{3} R}{6}+\frac{\left(\tilde{\rho}^{2}+2 V \tilde{\rho}\right)}{144 M^{6}}- \\
& -\left\{\frac{\mathcal{E}}{3}-\frac{1}{12 M^{3}}\left[\bar{\rho}-\frac{4}{3}\left(\bar{p}+T_{A B}^{\mathrm{BULK}} n^{A} n^{B}\right)\right]\right\}(33) \\
\dot{H} & =-H^{2}+\lambda-\frac{V(\tilde{\rho}+3 \tilde{p})}{144 M^{6}}-\frac{\left(2 \tilde{\rho}^{2}+3 \tilde{\rho} \tilde{p}\right)}{144 M^{6}}- \\
& -\left\{\frac{1}{12 M^{3}}\left[\bar{\rho}+\frac{4}{3}\left(\bar{p}+\frac{1}{2} T_{A B}^{\mathrm{BULK}} n^{A} n^{B}\right)\right]-\frac{\mathcal{E}}{3}\right\}(3)
\end{aligned}
$$

with $\mathcal{E}=\mathcal{E}_{\alpha \beta} \tilde{u}^{\alpha} \tilde{u}^{\beta}=C_{A C B D} \tilde{u}^{A} n^{C} \tilde{u}^{B} n^{D}$.

Employing the corresponding projection tensor $\bar{\Pi}_{A B}=$ $g_{A B}+\tilde{u}_{A} \tilde{u}_{B}-n_{A} n_{B}$ (projecting normally to $n^{A}$ and $\tilde{u}^{A}$ ), we decompose the isotropic pressure $\bar{p}$ into parallel and perpendicular parts according to

$$
\begin{gathered}
\bar{p}_{\|}=T_{A B}^{\mathrm{BULK}} n^{A} n^{B}, \quad p_{\perp}=\frac{1}{3} T_{A B}^{\mathrm{BULK}} \bar{\Pi}^{A B}, \quad \bar{p}=\frac{\bar{p}_{\|}+3 p_{\perp}}{4} \\
\bar{\rho}=-T^{\mathrm{BULK}}+\bar{p}_{\|}+3 p_{\perp} .
\end{gathered}
$$

We observe that $\bar{p}$ consists of two terms: a contribution $\bar{p}_{\|}$along the direction of $n^{A}$, and the perpendicular part $p_{\perp}$ that corresponds to the isotropic bulk pressure parallel to the $3 \mathrm{D}$ hypersurfaces $\mathcal{D}$. We note that, in a general brane, there will be an anisotropic bulk pressure described by the traceless part of $\bar{\Pi}_{A}^{C} \bar{\Pi}_{B}^{D} T_{C D}^{\mathrm{BULK}}$. However, for a FRW brane, because of the spatial homogeneity and isotropy of $\mathcal{D}$, the only pressure contribution coming from directions normal to both $\tilde{u}^{A}$ and $n^{A}$ is the isotropic part $p_{\perp}$.

Inserting equations (35) and (36) in (33) and (34) we obtain:

$$
\begin{gathered}
H^{2}=\lambda-\frac{{ }^{3} R}{6}+\frac{\left(\tilde{\rho}^{2}+2 V \tilde{\rho}\right)}{144 M^{6}}- \\
-\left\{\frac{\mathcal{E}}{3}-\frac{1}{12 M^{3}}\left[4 p_{\perp}-T^{\mathrm{BULK}}\right]\right\} \\
\dot{H}=-H^{2}+\lambda-\frac{V(\tilde{\rho}+3 \tilde{p})}{144 M^{6}}-\frac{\left(2 \tilde{\rho}^{2}+3 \tilde{\rho} \tilde{p}\right)}{144 M^{6}}- \\
-\left\{\frac{1}{12 M^{3}}\left[4 p_{\perp}+2 \bar{p}_{\|}-T^{\mathrm{BULK}}\right]-\frac{\mathcal{E}}{3}\right\} .
\end{gathered}
$$

Equations (37) and (38) show how the brane evolution is affected by the presence of matter in the bulk. For example, in the case of a general bulk perfect fluid (w.r.t. to the bulk observers $u^{A}$ ) the normal pressure $p_{\perp}$ is equal to the isotropic pressure $p$ measured by the bulk observers. Since $T^{\mathrm{BULK}}=-\rho+4 p=-\rho+4 p_{\perp}$, the last term in equation (37) becomes $\rho / 12 M^{3}$. The influence of the bulk fluid on the expansion arises through the local bulk matter density $\rho$ and the scalar $\mathcal{E}$ that can be loosely interpreted as accounting for the effect of the local gravitational field. In the case of a null radiation fluid (i.e. a generalized AdS-Vaidya bulk) the trace of the bulk energy-momentum tensor vanishes and the last term becomes $p_{\perp} / 3 M^{3}$.

Inserting equation (24) in (37) and (38) we get:

$$
\begin{aligned}
H^{2} \equiv \frac{\dot{\ell}}{\ell}=\lambda-\frac{{ }^{3} R}{6}+\frac{\left(\tilde{\rho}^{2}+2 V \tilde{\rho}\right)}{144 M^{6}}+\frac{\mathcal{M}}{6 \pi^{2} M^{3} \ell^{4}} \\
\dot{H}=-H^{2}+\lambda-\frac{V(\tilde{\rho}+3 \tilde{p})}{144 M^{6}}-\frac{\left(2 \tilde{\rho}^{2}+3 \tilde{\rho} \tilde{p}\right)}{144 M^{6}}- \\
-\frac{\mathcal{M}}{6 \pi^{2} M^{3} \ell^{4}}-\frac{1}{6 M^{3}} \bar{p}_{\| \cdot}
\end{aligned}
$$

We conclude that the total bulk contribution to the Friedmann equation is encompassed by the generalized comoving mass $\mathcal{M}$ of the bulk fluid, irrespectively of the dynamical interpetation with respect to the bulk observers.

Another intuitive reformulation of (39) and (40) is obtained by taking the derivative of the first equation along $\tilde{u}^{\alpha}$ and combining the result with the second one. We obtain the effective conservation equation

$$
[\dot{\tilde{\rho}}+3 H(\tilde{\rho}+\tilde{p})]\left(1+\frac{\tilde{\rho}}{V}\right)=-\left[\dot{\rho}_{\mathrm{eff}}+3 H\left(\rho_{\mathrm{eff}}+p_{\mathrm{eff}}\right)\right]
$$

where

$$
\rho_{\text {eff }}=\frac{12 M^{3}}{V} \frac{\mathcal{M}}{\pi^{2} \ell^{4}}, \quad p_{\text {eff }}=\frac{\rho_{\text {eff }}}{3}+\frac{8 M^{3}}{V} \bar{p}_{\|}
$$


can be regarded as the effective energy density and pressure arising through the bulk matter. The effective Friedmann equation (39) can be written as

$$
H^{2}=\lambda-\frac{{ }^{3} R}{6}+\frac{1}{6 M_{\mathrm{Pl}}^{2}}\left[\tilde{\rho}\left(1+\frac{\tilde{\rho}}{2 V}\right)+\rho_{\mathrm{eff}}\right],
$$

with $M_{\mathrm{Pl}}^{2}=12 M^{6} / V$. For low brane energy density $\tilde{\rho} \ll$ $V$, the terms $\sim \tilde{\rho}^{2}, \tilde{\rho} \tilde{p}$ become negligible.

\section{CONCLUSIONS}

Our main results are summarized by equations (39)(43). The first equation indicates that the effect of the bulk matter on the Friedmann equation is incorporated in the generalized comoving mass $\mathcal{M}$ of the bulk fluid, irrespectively of the nature of this fluid. This is reminiscent of the implications of Birkhoff's theorem for the gravitational field generated by a matter distribution. Both results are consequences of the assumed Local Rotational Symmetry of the geometry that is inherited by the matter distribution (through the assumed symmetry inheritance by the bulk observers).

The equivalent form (43) of the Friedmann equation indicates that the bulk generates an effective matter component that contributes to the brane cosmological evolution. For low brane energy densities two fluids contribute linearly to the expansion: The brane matter with energy density $\tilde{\rho}$, and the effective (or "mirage") matter with energy density $\rho_{\text {eff }}$ given by the first of equations (42). A remarkable conclusion can be drawn from equation (41) for low brane energy densities: There is conservation of energy between the two components. The brane matter scales as a perfect fluid with an equation of state $\tilde{p}=\tilde{p}(\tilde{\rho})$. The mirage component again scales as a perfect fluid with an effective pressure given by the second of equations (42). Energy exchange is possible between the two fluids. We have verified that equations (39)- (43) reproduce successfully the known cosmological evolution in a variety of models for the bulk geometry (Schwarzschild-
AdS [4], Vaidya-AdS 8, 9], static perfect fluid in an AdS bulk [13], etc).

The equation of state $p_{\text {eff }}=p_{\text {eff }}\left(\rho_{\text {eff }}\right)$ of the mirage component is not expected in general to have a simple form. The reason is that $\bar{p}_{\|}$is the pressure of the bulk fluid perpendicularly to the brane, as measured by the brane observer. The only simple case involves an empty bulk energy-momentum tensor, for which $\bar{p}_{\|}=0, p_{\text {eff }}=$ $\rho_{\text {eff }} / 3$. This is the well known case of mirage radiation with $\rho_{\text {eff }}=12 M^{3} \mathcal{M}_{0} /\left(\pi^{2} V \ell^{4}\right)$.

An interesting question concerns the possibility of having accelerated expansion on the brane as a result of the brane-bulk interaction. The Raychaudhuri equation (40) provides important intuition on this problem in a general framework. The acceleration parameter is proportional to $\dot{H}+H^{2}$. For this to be positive one or more of the following conditions must be satisfied: a) The effective cosmological constant $\lambda$ is positive. b) The brane matter satisfies $\tilde{\rho}<V$ and $\tilde{p}<-\tilde{\rho} / 3$. c) The brane matter satisfies $\tilde{\rho}>V$ and $\tilde{p}<-2 \tilde{\rho} / 3$. d) The comoving mass $\mathcal{M}$ is negative. e) The pressure $\bar{p}_{\|}$of the bulk fluid perpendicularly to the brane, as measured by the brane observer, is negative. In general negative pressures are associated with field configurations. The possibility of a negative comoving mass seems problematic at first sight, as usually it implies the existence of naked singularities or instabilities. A counter example is a brane with negative tension in the two-brane model of 1 ].

\section{ACKNOWLEDGMENTS}

One of the authors (NT) is partially supported through the RTN contract MRTN-CT-2004-503369 of the European Union and the research program "Kapodistrias" of the University of Athens. Both authors acknowledge the financial support of Ministry of National Education through the research program "Pythagoras", grant no 70-03-7310.
[1] L. Randall and R. Sundrum, Phys. Rev. Lett. 83 (1999) 3370 arXiv:hep-th/9905221;

Phys. Rev. Lett. 83 (1999) 4690 arXiv:hep-th/9906064.

[2] P. Binetruy, C. Deffayet and D. Langlois, Nucl. Phys. B 565 (2000) 269 arXiv:hep-th/9905012;

P. Binetruy, C. Deffayet, U. Ellwanger and D. Langlois, Phys. Lett. B 477 (2000) 285 arXiv:hep-th/9910219.

[3] C. Csaki, M. Graesser, C. F. Kolda and J. Terning, Phys. Lett. B 462 (1999) 34 arXiv:hep-ph/9906513;

J. M. Cline, C. Grojean and G. Servant, Phys. Rev. Lett. 83 (1999) 4245 arXiv:hep-ph/9906523.

[4] P. Kraus, JHEP $\mathbf{9 9 1 2}$ (1999) 011 arXiv:hep-th/9910149.

[5] T. Shiromizu, K. i. Maeda and M. Sasaki, Phys. Rev. D
62, 024012 (2000) arXiv:gr-qc/9910076.

[6] P. Brax and C. van de Bruck, Class. Quant. Grav. 20 (2003) R201 arXiv:hep-th/0303095;

[7] R. Maartens, Living Rev. Rel. 7, 7 (2004) gr-qc 0312059.

[8] A. Hebecker and J. March-Russell, Nucl. Phys. B 608 (2001) 375 arXiv:hep-ph/0103214;

D. Langlois, L. Sorbo and M. Rodriguez-Martinez, Phys. Rev. Lett. 89 (2002) 171301 arXiv:hep-th/0206146; E. Leeper, R. Maartens and C. F. Sopuerta, Class. Quant. Grav. 21 (2004) 1125 arXiv:gr-qc/0309080.

[9] N. Tetradis, Class. Quant. Grav. 21 (2004) 5221 arXiv:hep-th/0406183.

[10] H. A. Chamblin and H. S. Reall, Nucl. Phys. B 562 (1999) 133 arXiv:hep-th/9903225; 
C. Barcelo and M. Visser, Phys. Rev. D 63 (2001) 024004 arXiv:gr-qc/0008008;

K. i. Maeda and D. Wands, Phys. Rev. D 62 (2000) 124009 arXiv:hep-th/0008188;

A. Mennim and R. A. Battye, Class. Quant. Grav. 18 (2001) 2171 arXiv:hep-th/0008192.

[11] C. van de Bruck, M. Dorca, C. J. A. Martins and M. Parry, Phys. Lett. B 495 (2000) 183 arXiv:hep-th/0009056.

[12] E. Kiritsis, G. Kofinas, N. Tetradis, T. N. Tomaras $\begin{array}{lllll}\text { and V. Zarikas, JHEP } & 0302 & \text { (2003) } & 035\end{array}$ arXiv:hep-th/0207060;

N. Tetradis, Phys. Lett. $\quad$ B $\quad \mathbf{5 6 9} \quad$ (2003) 1 arXiv:hep-th/0211200.

[13] P. S. Apostolopoulos and N. Tetradis, Class. Quant.
Grav. 21 (2004) 4781 arXiv:hep-th/0404105.

[14] H. van Elst and G. F. R. Ellis, Class. Quant. Grav. 13 (1996) 1099 gr-qc/9510044.

[15] G. F. R. Ellis, Cosmological Models in Cargèse Lectures (1998) gr-qc/9812046.

[16] W. Israel, Nuovo Cim. B 44 (1966) 1.

[17] D. Birmingham, Class. Quant. Grav. 16 (1999) 1197 arXiv:hep-th/9808032.

[18] S. Mukohyama, T. Shiromizu and K. i. Maeda, Phys. Rev. D 62 (2000) 024028 [Erratum-ibid. D 63 (2001) 029901] arXiv:hep-th/9912287;

P. Bowcock, C. Charmousis and R. Gregory, Class. Quant. Grav. 17 (2000) 4745 arXiv:hep-th/0007177. 